\title{
Study of Heavy Quarkonium with Energy Dependent Potential
}

\author{
Pramila Gupta, Indira Mehrotra \\ Nuclear and Particle Physics Group, Department of Physics, University of Allahabad, Allahabad, India \\ Email: pramila62@gmail.com
}

Received August 10, 2012; revised September 9, 2012; accepted September 17, 2012

\begin{abstract}
Heavy quark systems ( $c \bar{c}$ and $b \bar{b})$ have been studied in the nonrelativistic framework using energy dependent interquark potential of the form harmonic oscillator with a small linear term as energy dependent as perturbation plus a inverse square potential. This potential admits exact analytical solution of the Schrodinger equation. Mass spectra, leptonic decay width, root mean square radii $\left(\sqrt{\left\langle r^{2}\right\rangle}\right)$, the expectation value of the radius $(\langle r\rangle)$ and $\langle 1 / r\rangle$ have been estimated for different quantum mechanical states for $c \bar{c}$ and $b \bar{b}$ systems. It is observed that energy dependent term in the potential leads to saturation of the mass spectra and degree of saturation is governed by the magnitude of perturbation. The calculated values of leptonic decay widths for 1s state are in very good agreement with the experimental data both for $c \bar{c}$ and $b \bar{b}$ systems.
\end{abstract}

Keywords: Heavy Quark; Mass Spectrum; Energy Dependent Potential

\section{Introduction}

Energy spectrum of heavy quarkonium are a rich source of information on the nature of the interquark force at distances $<0.1 \mathrm{fm}$ and $>1.0 \mathrm{fm}$. Many features such as mass spectra and decay properties of heavy quarkonia could be described by applying the ordinary nonrelativistic Schrödinger wave equation to the two body quarkantiquark system. It is well known that the non-relativistic approach is justified when large quark masses are involved and level spacing between the energy levels is less than the constituent masses.

A variety of forms for the interquark potential have been used in heavy quarkonia mass spectroscopy. These can be broadly classified as 1) QCD motivated potential [1-5] and 2) purely phenomenological potential [6-9]. A comprehensive list of potential models is described in the work of Lichtenberg [10]. All the potentials have almost similar behavior in the range of $0.1 \mathrm{fm} \leq r \leq 0.1 \mathrm{fm}$, the characteristic interval of $c \bar{c}$ and $b \bar{b}$ systems but differ from each other outside this range. At present it is not possible to obtain exact interquark potential in the entire range of distances from the first principal of Quantum Chromodynamics. Moreover major shortcoming of the existing potentials is that they have not been able to account for the observed saturation pattern in the experimental spectra.
A different category of potentials, which are energy dependent, have been known in physics for a long time. They occur in relativistic quantum mechanics at various places like with Pauli Schrodinger equation [11], in the Hamiltonian formulation of relativistic many body problem in covariant formulation with constraints in nonlinear Hamiltonian evolution equation, and also in soliton propagation. In the non-relativistic physics energy dependent potentials offer the possibility of studying nonlinear effects in the framework of Schrodinger equation. Lombard [12] have for the first time used an energy dependent potential to study the bound state properties of $c \bar{c}$ and $b \bar{b}$ systems. Initially they had used one dimensional harmonic oscillator potential with a linear energy dependent term as perturbation to study the effect of energy dependence on the energy eigenvalues. Later they solved the problem for three dimensions with different power law potentials (harmonic, linear and Coulomb) with energy dependence. Their main conclusion has been that energy dependence saturates the mass spectra. It is well known that any realistic interquark potential has two components: asymptotic and confinement. However Lombard's potential has only one component either confinement (harmonic and linear) or asymptotic (Coulomb). In view of this in the present work we have used a more realistic interquark potential of the form harmonic plus inverse square with small linear energy 
dependence on the confining harmonic oscillator potential. The choice of linear dependence is motivated by the fact that it leads to a coherent theory [13]. The combination of harmonic oscillator and inverse square potential was first of all adopted by Joshi and Mitra [14] in a theory based on the Schrodinger equation for studying the heavy meson spectroscopy. Later it has been used by Iyer et al. [15] and Ryes et al. [16] in the study of hadron spectroscopy. In all these studies a better fit was obtained for the potential with a term proportional to $1 / r^{2}$ because it has singularity for $r \rightarrow 0$ that improves the behavior in this region. Also one great advantage is that our potential admits exact analytical solution for the radial part of the Schrodinger equation. This is a great advantage in view of the high nonlinearity of the differential equation to be solved. Using this potential we have calculated the mass spectrum, the root mean square radii, average radii, leptonic decay width and $\langle 1 / r\rangle$ for $c \bar{c}$ and $b \bar{b}$ systems. The latter two properties are sensitive to the asymptotic part of the potential. The aim is to study the effect of energy dependence on the low as well as high excitation states of the system.

\section{Details of Calculation}

In the present work, energy spectrum of heavy quarkonium systems ( $c \bar{c}$ and $b \bar{b})$ have been studied in the framework of non-relativistic Schrödinger equation using interquark potential as spin independent harmonic oscillator with a small linear term energy dependent plus inverse square potential given by

$$
V\left(r, E_{n, l}\right)=\frac{1}{2} m \omega^{2} r^{2}\left(1+\gamma E_{n, l}\right)+\frac{g}{r^{2}},
$$

where $\omega, g$ and $\gamma$ are constants.

The three dimensional Schrodinger equation in the center-of-mass system is

$$
\left[-\frac{\hbar^{2}}{2 \mu} \nabla^{2}+V\left(r, E_{n, l}\right)\right] \psi_{n, l, m}(r, \theta, \phi)=E_{n, l} \psi_{n, l, m}(r, \theta, \phi),
$$

where the reduced mass $\mu$ in terms of quark mass $m_{q}$ and antiquark mass $m_{q}$ is

$$
\mu=\frac{m_{q} m_{\bar{q}}}{m_{q}+m_{\bar{q}}} .
$$

In natural units $\hbar=c=1$ is considered.

The wave function is written as

$$
\psi_{n, l, m}(r, \theta, \phi)=\frac{u_{n, l}(r)}{r} Y_{l, m}(\theta, \phi)
$$

Now putting $\omega^{\prime 2}=\omega^{2}(1+\gamma E), t=m \omega^{\prime} r^{2}, \quad \alpha=\frac{2 E}{\omega^{\prime}}$ and $\beta=l(l+1)+2 \mu g$, Equation (2) reduces to

$$
t u^{\prime \prime}(t)+\frac{u^{\prime}(t)}{2}+\frac{1}{4}\left[\alpha-\frac{\beta}{t}-t\right] u(t)=0
$$

$u(t) \mathrm{s}$ are the solution of the radial equation, which are bounded at infinity and are zero at the origin. As $t$ tends to $\infty$, the bounded solution behaves like $\exp (-t / 2)$ and since $t=0$ is a singularity of Equation (3) we seek for a solution in the form,

$$
u(t)=t^{\eta} \exp \left(\frac{-t}{2}\right) R_{l}(t)
$$

in which, on account of boundary condition, $\eta$ has to be positive. Substituting Equation (4) into Equation (3) and taking

$$
\eta=\frac{1}{4}\left(1+\sqrt{(2 l+1)^{2}+8 \mu g}\right)
$$

This leads to the equation

$$
t R_{l}^{\prime \prime}(t)+\left[2 \eta+\frac{1}{2}-t\right] R_{l^{\prime}}^{\prime}(t)-\left[\eta+\frac{1}{4}-\frac{\alpha}{4}\right] R_{l}(t)=0
$$

Apart from the constant factor the nonsingular solution of Equation (6) is the Confluent Hypergeometric series [17]

$$
R_{l}(t)=F\left[\eta+\frac{1}{4}-\frac{\alpha}{4}, 2 \eta+\frac{1}{2} ; t\right] .
$$

$u(t)$ increases without bound as $t \rightarrow \infty$ unless the series $F$ reduces to a polynomial. This occurs only if

$$
\eta+\frac{1}{4}-\frac{\alpha}{4}=-n_{r} ; n_{r}=0,1,2 \cdots,
$$

which implies that the energy eigenvalue

$$
E_{n, l}=-\frac{1}{8} a^{2} \omega^{2} \gamma+\frac{a \omega}{8} \sqrt{a^{2} \omega^{2} \gamma^{2}+16}
$$

where $a=4 n-2+\sqrt{(2 l+1)^{2}+8 \mu g}$.

$E_{n, l}$ is the classified eigen energy by principal quantum number and angular quantum number $\ell \leq(n-1)$ and the quark mass is connected to the physical mass as $M_{(q q)}=2 m_{q}+E_{1 s}$.

The parameters $\omega, g$ and $\gamma$ are obtained from fit to the experimental energies of triplet states of $2 \mathrm{~s}$ and $3 \mathrm{~s}$ and center of gravity of $1 \mathrm{p}$ with respect to $E_{1 S}$ in Equation (9). In the case of energy independent potential $(\gamma=$ 0 ) the two parameters $\omega$ and $g$ are obtained from fitting to the theoretically estimated values of $2 \mathrm{~s}$ and $1 \mathrm{p}$ to the corresponding experimental data. These in turn are used to predict eigenvalues of higher excited levels from the energy eigenvalue Equation (9). All the experimental data for $c \bar{c}$ and $b \bar{b}$ are taken from recent compilation of Particle Data Group 2008 [18]. In the literature, the charm quark mass is chosen between $1.2<m_{c}<1.8 \mathrm{GeV}$ 
whereas that of the bottom quark is between $4.5<m_{b}<$ $5.4 \mathrm{GeV}$. In the present work we have chosen the mass to be $1.5 \mathrm{GeV}$ for the charm quark and $5.0 \mathrm{GeV}$ for the bottom quark, which is almost at the mid values of above ranges. While solving non linear energy eigenvalue Equation (9) only negative roots of $\gamma$ are accepted because negative values of $\gamma$ can compress the spectrum which is experimentally observed. Once the parameters of the potential are fixed it is substituted in the reduced radial Schrodinger equation given by

$$
u_{n, l}^{\prime \prime}(r)=2 \mu\left[V\left(r, E_{n, l}\right)+\frac{l(l+1)}{2 \mu r^{2}}-E_{n, l}\right] u_{n, l}(r)
$$

This equation is solved numerically in MATHEMATICA 8.0 by software program obtained by Lucha et $a l$. [19] for each quantum state separately. The exact numerical solution of wave functions so obtained are used to calculate the leptonic decay width, $\langle r\rangle,\langle 1 / r\rangle$ and $\sqrt{\left\langle r^{2}\right\rangle}$.

Vector state with spin one, negative parity $\left({ }^{3} S_{1}\right)$ of quark antiquark pair can annihilate into lepton pair through single virtual photon. Leptonic decay width of ${ }^{3} S_{1}$ states of $c \bar{c}$ and $b \bar{b}$ quarkonia is the physical quantity which is very sensitive to the form of potential. Leptonic decay widths are calculated according to Van Royen-Weisskoph formula [20]. This formula is true for energy dependent potential also.

$$
\Gamma\left(n^{3} S_{1} \rightarrow e^{+} e^{-}\right)=\frac{16 \pi \alpha^{2} e_{q}^{2}}{M_{n s}^{2}(q \bar{q})}\left|\psi_{n, s}(0)\right|^{2}\left(1-\frac{16 \alpha_{s}\left(m^{2} q\right)}{3 \pi}\right)
$$

where $\left|\psi_{n S}(0)\right|$ is the bound state radial wave function of s wave at the origin. $M_{n s}$ is the mass of bound triplet (vector) state, $\alpha$ is the electromagnetic fine structure constant and $e_{q}$ the charge of quark in units of the electron charge $e_{c}=(2 / 3) e, e_{b}=-(1 / 3) e$.

In the present work we have taken strong coupling constants as $a_{s}\left(m_{c}^{2}\right)=0.37$ and $a_{s}\left(m_{b}^{2}\right)=0.26$.

Expectation values of $r, 1 / r$ and bound state root mean square radii (rms) are obtained from

$$
\langle x\rangle=\frac{\int_{0}^{\infty} x u^{2}(r) \mathrm{d} r}{\int_{0}^{\infty} u^{2}(r) \mathrm{d} r},
$$

where $x$ stands for $r, 1 / r$ and $r^{2}$.

\section{Results and Discussion}

The potential parameters obtained from fit to experimental energy levels are given for $c \bar{c}$ and $b \bar{b}$ systems in Tables 1 and 2 respectively. These exhibit flavor dependence. Parameters of set A correspond to energy independent potential and are compared with the parameters obtained by Ryes et al. [16] for harmonic oscillator plus inverse square potential. Parameters of set $\mathrm{B}$ are for energy dependent potential used in present work. These are compared with the values obtained by Lombard et al. [12] for an energy dependent harmonic oscillator potential. The quark masses used are slightly different in each work.

The corresponding potentials as a function of distance are plotted in Figures 1 and 2. The energy dependent perturbation leads to slight variation of confinement potential for different states. When the energy of the state increases with radial excitation, the classically allowed region for harmonic oscillator potential is greater.

Table 1. Spectroscopic parameters obtained from fit to experimental data for $c \bar{c}$ system.

\begin{tabular}{ccccc}
\hline Parameters & Present work (A) & Ref [16] & Present work (B) & Ref [12] \\
\hline$m_{q}(\mathrm{GeV})$ & 1.50 & 3.812 & 1.50 & 1.207 \\
$\gamma\left(\mathrm{GeV}^{-1}\right)$ & 0 & 0 & -0.117 & -0.433 \\
$\omega(\mathrm{GeV})$ & 0.174 & 0.285 & 0.203 & 0.55 \\
$g\left(\mathrm{GeV}^{-1}\right)$ & -0.073 & -0.0655 & -0.155 & 0 \\
\hline
\end{tabular}

Table 2. Spectroscopic parameters obtained from fit to experimental data for $b \bar{b}$ system.

\begin{tabular}{ccccc}
\hline Parameters & Present work (A) & Ref [16] & Present work (B) & Ref [12] \\
\hline$m_{q}(\mathrm{GeV})$ & 5.00 & 7.093 & 5.00 & 4.401 \\
$\gamma\left(\mathrm{GeV}^{-1}\right)$ & 0 & 0 & -0.102 & -0.455 \\
$\omega(\mathrm{GeV})$ & 0.176 & 0.2090 & 0.187 & 0.530 \\
$g\left(\mathrm{GeV}^{-1}\right)$ & -0.0425 & -0.0352 & -0.044 & 0 \\
\hline
\end{tabular}




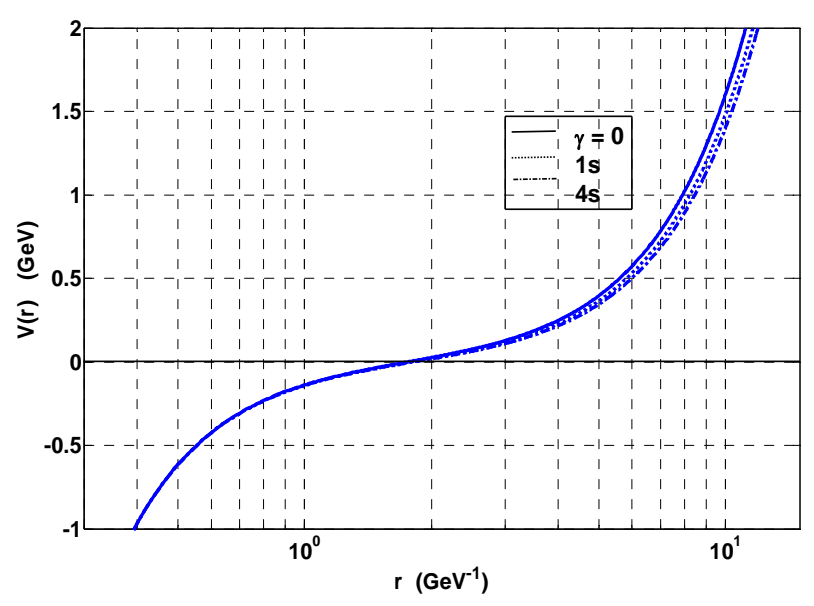

Figure 1. Quark interquark potential curves as a function of $r$ for energy dependent (1s and $4 \mathrm{~s}$ states) and energy independent case $(\gamma=0)$ for $c \bar{c}$.

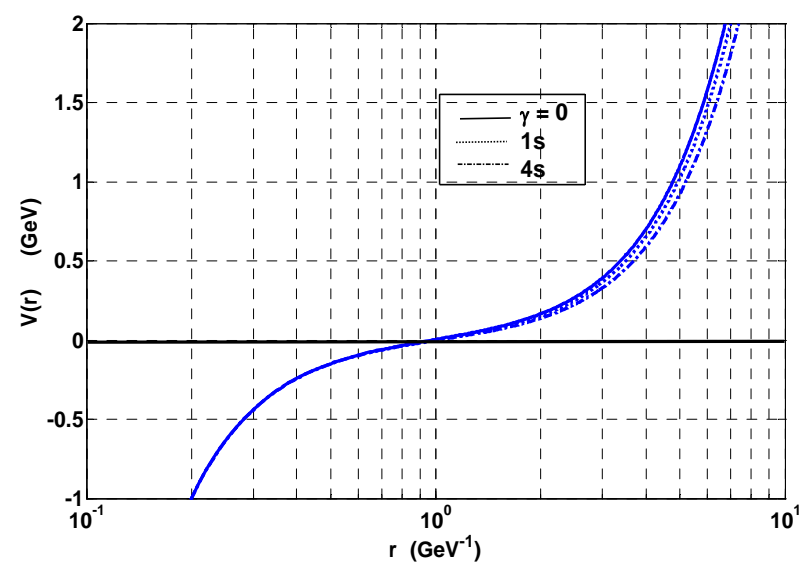

Figure 2. Quark interquark potential curves as a function of $r$ for energy dependent (1s and $4 \mathrm{~s}$ states) and energy independent case $(\gamma=0)$ for $b \bar{b}$.

The energy spectra of charmonium and bottonium system with respect to $E_{1 s}$ are shown in Figures 3 and 4 respectively. We compare our plotted mass spectra results with and without energy dependence with experimental data and also with the work of Lombard et al. [12].

Position of different ns states with respect to $1 \mathrm{~s}$ is shown in Table 3. It is observed that the $E_{n, s}-E_{1, s}$ increases slowly with principal quantum number. This increase is less rapid for energy dependent potential, showing saturation effect as compared to energy independent case $(\gamma=0)$. The eigenvalues $E_{1 \ell}$ are displayed up to $\ell=20$ for different values of $\gamma$ in Figure 5 for the $c \bar{c}$ system. The interesting feature of the result is that $\gamma$ determines the level of saturation. On decreasing $\gamma$, maximum value of eigenvalue $E_{1 \ell}$ decreases and reaches an upper limit. In contrast for energy independent case $\mathrm{E}_{1 \ell}$ increases regularly towards with increasing $\ell$. Similar plot up to $\ell=30$ is shown for the $b \bar{b}$ system in

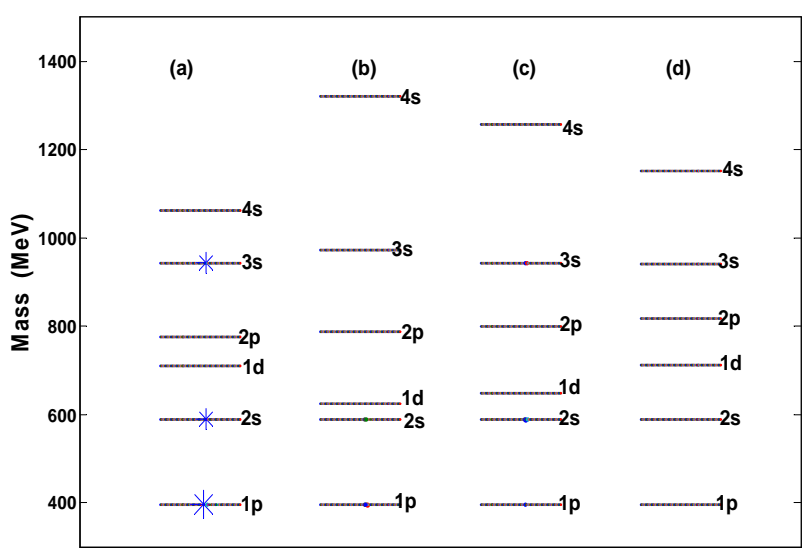

Figure 3. Mass spectrum of charmonium system with respect to ground state $\left(E_{1 s}\right)$ with (a) experimental data (b) $\gamma=$ 0 (c) $\gamma \neq 0$ (d) Lombard's work. The levels with asterisk are used as input data in the parameter fitting.

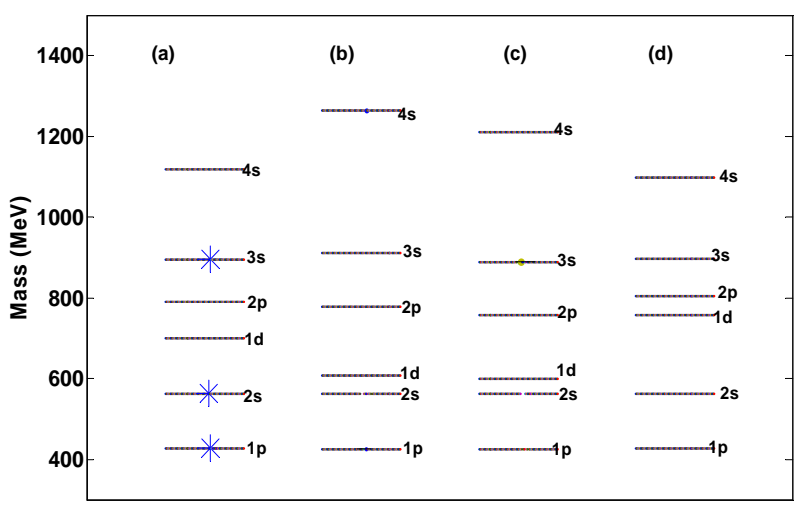

Figure 4. Mass spectrum of bottonium system with respect to ground state $\left(E_{1 s}\right)$ with (a) experimental data (b) $\gamma=0$ (c) $\gamma \neq 0$ (c) Lombard's work. The levels with asterisk are used as input data in the parameters fitting.

Table 3. Spacing between the various radial excitations for $\ell$ $=0$.

\begin{tabular}{lcccc}
\hline & \multicolumn{2}{c}{$c \bar{c}$} & \multicolumn{2}{c}{$b \bar{b}$} \\
\cline { 2 - 5 } & $\gamma=0$ & $\gamma \neq 0$ & $\gamma=0$ & $\gamma \neq 0$ \\
\hline$E_{45}-E_{1 S}(\mathrm{GeV})$ & 1.25 & 1.20 & 1.26 & 1.21 \\
$E_{55}-E_{1 S}(\mathrm{GeV})$ & 1.65 & 1.61 & 1.61 & 1.57 \\
$E_{65}-E_{1 S}(\mathrm{GeV})$ & 1.98 & 1.96 & 1.97 & 1.95 \\
\hline
\end{tabular}

Figure 6. The saturation in $b \bar{b}$ is reached more slowly compared to $c \bar{c}$ system. We have studied the effect of varying quark mass on the value of the parameters for $c \bar{c}$ and $b \bar{b}$ systems. It turns out that all the parameters vary with quark mass. The variation of $g$ with $m_{c}$ and $m_{b}$ is given in Table 4 keeping $\gamma$ and $\omega$ constant.

Leptonic decay width of vector meson for 1s state and its ratio with those of other states are listed and also compared with experimental data in Table 5. Leptonic 


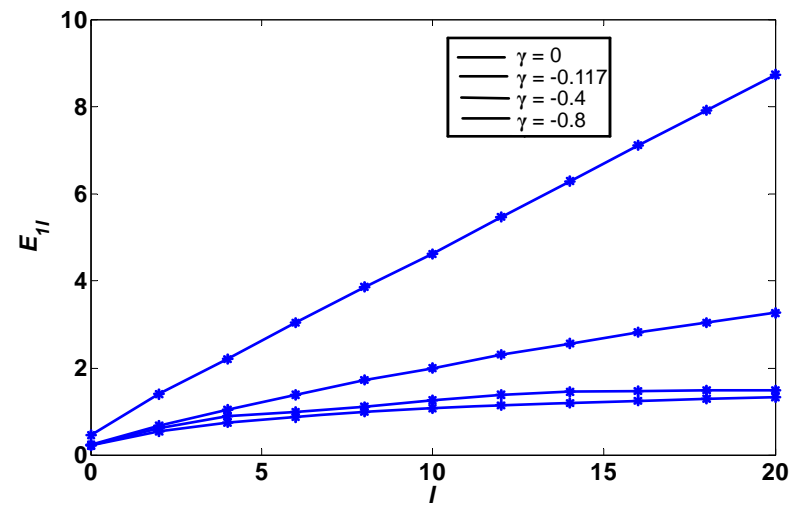

Figure 5. Behavior of spectrum for different values of $\gamma$ in $c \bar{c}$ system.

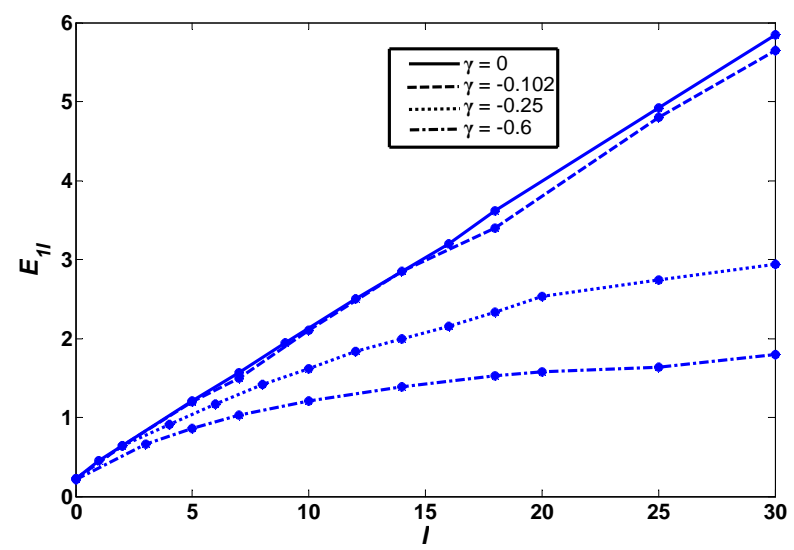

Figure 6. Behavior of spectrum for different values of $\gamma$ in b $\bar{b}$ system.
Table 4. Values of parameter $\mathrm{g}$ for different masses of $c \bar{c}$ and $b \bar{b}$ system.

\begin{tabular}{cccc}
\hline \multicolumn{2}{c}{$c \bar{c}$} & \multicolumn{2}{c}{$b \bar{b}$} \\
\hline Mass $(\mathrm{GeV})$ & $\mathrm{g}\left(\mathrm{GeV}^{-1}\right)$ & Mass $(\mathrm{GeV})$ & $\mathrm{g}\left(\mathrm{GeV}^{-1}\right)$ \\
\hline 1.2 & -0.194 & 4.4 & -0.045 \\
1.5 & -0.155 & 5.0 & -0.040 \\
1.7 & -0.130 & 5.5 & -0.036 \\
\hline
\end{tabular}

decay width for 1s state obtained in the present work are very close to experimental data and almost double of the value of obtained by Lombard. This shows the importance of using asymptotic term in the potential.

Results for $\langle r\rangle,\langle 1 / r\rangle$ and $\sqrt{\left\langle r^{2}\right\rangle}$ computed for different quantum states of $c \bar{c}$ and $b \bar{b}$ systems are listed in Tables 6 and 7. Also given for comparison are the results of the calculation of Boroum et al. [21] for $\langle r\rangle$ and $\langle 1 / r\rangle$ with global potential and of Chen Hong et al [22] for root mean square radius with QCD based potential. Root mean square radii for different $1 \mathrm{~s}$ state have slightly smaller values as compared with Lombard potential reported as 0.49 (for $c \bar{c}$ ) and 0.26 (for $b \bar{b}$ ). Our estimated values fall well in the range of the results of other calculation.

It is observed that $b \bar{b}$ system has smaller radii than the $c \bar{c}$ system. Root mean square radii of various states of $c \bar{c}$ and $b \bar{b}$ fall within the interval 0.1 to $1 \mathrm{fm}$ because all the potentials are similar in this range. It means that average size (radius) of the $c \bar{c}$ system is greater

Table 5. Ratio of Leptonic decay width of different states with that of $1 \mathrm{~s}$ state.

\begin{tabular}{ccccc}
\hline & \multicolumn{3}{c}{$c \bar{c}$} & $b \bar{b}$ \\
\cline { 2 - 5 } & Calculated value & Experimental value [18] & Calculated value & Experimental value [18] \\
\hline$\Gamma(1 \mathrm{~s})(\mathrm{kev})$ & $5.15(2.65)$ & $5.55 \pm 0.14$ & $1.05(0.47)$ & $1.32 \pm 0.018$ \\
$\Gamma_{e^{+} e^{+}}(2 \mathrm{~s}) / \Gamma_{e^{+} e^{-}}(1 \mathrm{~s})$ & 0.61 & $0.45 \pm 0.08$ & 0.68 & $0.46 \pm 0.03$ \\
$\Gamma_{e^{+} e^{+}}(3 \mathrm{~s}) / \Gamma_{e^{+} e^{-}}(1 \mathrm{~s})$ & 0.50 & $0.16 \pm 0.04$ & 0.63 & $0.33 \pm 0.03$ \\
$\Gamma_{e^{+} e^{+}}(4 \mathrm{~s}) / \Gamma_{e^{+} e^{-}}(1 \mathrm{~s})$ & 0.43 & $0.11 \pm 0.04$ & 0.57 & $0.23 \pm 0.02$ \\
\hline
\end{tabular}

Table 6. $\langle r\rangle,\langle 1 / r\rangle$ and $\sqrt{\left\langle r^{2}\right\rangle}$ for $c \bar{c}$.

\begin{tabular}{ccccccc}
\hline & \multicolumn{2}{c}{$\langle r\rangle\left(\mathrm{GeV}^{-1}\right)$} & \multicolumn{2}{c}{$\langle 1 / r\rangle(\mathbf{G e V})$} & \multicolumn{2}{c}{$\sqrt{\left\langle r^{2}\right\rangle}(\mathbf{f m})$} \\
\cline { 2 - 7 } & Present work & {$[21]$} & Present work & {$[21]$} & Present work & {$[22]$} \\
\hline $1 \mathrm{~s}$ & 2.790 & 2.618 & 0.507 & 0.491 & 0.456 & 0.401 \\
$2 \mathrm{~s}$ & 4.612 & 4.761 & 0.396 & 0.325 & 0.898 & 0.801 \\
$3 \mathrm{~s}$ & 5.9 & & 0.343 & & 1.252 & 1.242 \\
$1 \mathrm{p}$ & 4.266 & 3.751 & 0.316 & 0.307 & 0.698 & 0.639 \\
$2 \mathrm{p}$ & 5.588 & & 0.239 & & 1.113 & 1.101 \\
\hline
\end{tabular}


Table 7. $\langle r\rangle,\langle 1 / r\rangle$ and $\sqrt{\left\langle r^{2}\right\rangle}$ for $b \bar{b}$.

\begin{tabular}{ccccccc}
\hline & \multicolumn{2}{c}{$\langle r\rangle\left(\mathrm{GeV}^{-1}\right)$} & \multicolumn{2}{c}{$\langle 1 / r\rangle(\mathrm{GeV})$} & \multicolumn{2}{c}{$\sqrt{\left\langle r^{2}\right\rangle}(\mathbf{f m})$} \\
\cline { 2 - 7 } & Present work & {$[21]$} & Present work & {$[21]$} & Present work & {$[22]$} \\
\hline $1 \mathrm{~s}$ & 1.574 & 1.823 & 0.8706 & 0.685 & 0.206 & 0.196 \\
$2 \mathrm{~s}$ & 2.523 & 3.100 & 0.6946 & 0.486 & 0.510 & 0.490 \\
$3 \mathrm{~s}$ & 3.207 & & 0.6701 & & 0.790 & 0.781 \\
$1 \mathrm{p}$ & 2.306 & 2.446 & 0.4927 & 0.467 & 0.415 & 0.395 \\
$2 \mathrm{p}$ & 3.017 & & 0.44212 & & 0.715 & 0.693 \\
\hline
\end{tabular}

than the $b \bar{b}$ system i.e. heavy quarkonium have smaller radii.

\section{Summary and Conclusion}

Heavy quarkonia system (and $b \bar{b}$ ) have been studied in the framework of non-relativistic Schrodinger equation with energy dependent potential. Such potentials constitute a way to include nonlinear effects in the Schrodinger equation. The interquark potential used in the present work is of harmonic oscillator plus inverse square form with a small energy dependent term in harmonic oscillator part. This potential is more realistic compared to the energy dependent harmonic oscillator potential used in the work of Lombard which does not have the asymptotic term. The energy dependence in the potential results in the compression of the spectrum. As the quantum numbers increase the energy eigenvalues increase an upper bound. Though the choice of potential parameters is not unique, the above form represents a class of potentials which can give a satisfactory explanation of the saturation pattern in the spectra as well as the correct order of magnitude of Leptonic decay widths both for $c \bar{c}$ and $b \bar{b}$ systems. Our results for the Leptonic decay width of 1s state $\left(\Gamma_{1 s}\right)$ are much closer to the experimental values compared to those obtained by Lombard (values given within bracket in Table 5) for both $c \bar{c}$ and $b \bar{b}$ systems. This shows the importance of the asymptotic term in the interquark potential in determining the wavefunction at the origin.

\section{REFERENCES}

[1] E. Eichten, K. D. Lane, et al., "Spectrum of Charmed Quark Antiquark Bound States," Physical Review Letters, Vol. 34, No. 6, 1975, pp. 369-372. doi:10.1103/PhysRevLett.34.369

[2] J. L. Richarson, "The Heavy Quark Potential $J / \psi$ and Systems," Physics Letters B, Vol. 82, 1979, p. 272.

[3] W. Buchmuller, "Quarkonia and Quantum Chromodynamics," Physical Review Letters, Vol. 45, 1980, p. 403.

[4] S. N. Gupta and E. F. Radford, "Quarkonium spectra and Quantum Chromodynamics," Physical Review D, Vol. 26,
No. 11, 1982, pp. 3305-3308.

doi:10.1103/PhysRevD.26.3305

[5] K. Igi and S. Ono, "Heavy-Quarkonium Systems and the QCD Scale Parameter," Physical Review D, Vol. 33, No. 11, 1986, pp. 3349-3357. doi:10.1103/PhysRevD.33.3349

[6] C. Quigg and J. L. Rosner, "Realizing the Potential of Quarkonium," Physics Report C, Vol. 56, 1979, p. 167.

[7] A. Martin, "A Simultaneous Fit of $b b, c s, s s$ (bcs Pairs) and cs Spectra," Physics Letter B, Vol. 100, 1981, p. 511.

[8] A. K. Rai, B. Patel and P. C. Vinodkumar, "Properties of $q q$ Meson in Nonrelativistic QCD Formulism," Physical Review C, Vol. 78, No. 5, 2008, Article ID: 055202. doi:10.1103/PhysRevC.78.055202

[9] Bhaghyesh, K. B. V. Kumar and A. P. Monteiro, "Heavy Quarkonium Spectra and Its Decays in a Nonrelativistic Model with Hulthen Potential," Journal of Physics G: Nuclear and Particle Physics, Vol. 38, 2011, Article ID: 085001. doi:10.1088/0954-3899/38/8/085001

[10] D. B. Lichtenberg, "Energy Levels of Quarkonia in Potential Models," International Journal of Modern Physics A, Vol. 2, 1987, p. 1669.

[11] G. F. T. del Castillo and J. V. Castro, "Schrodinger-Pauli Equation for Spin-3/2 Particles," Resista Mexicana de Fisica, Vol. 50, No. 3, 2004, pp. 306-310.

[12] R. J. Lombard, J. Mars and C. Volpe, "Wave Equations of Energy Dependent Potentials for Confined Systems," Journal of Physics G: Nuclear and Particle Physics, Vol. 34, 2007, pp. 1879-1888.

[13] J. Formanck, R. J. Lombard and J. Mares, "An Extended Scenario for the Schrodinger Equation," Czechoslovak Journal of Physics, Vol. 54, 2006, p. 289.

[14] G. C. Joshi and A. N. Mitra, "Centrifugal and Harmonic Oscillator Potential for Heavy Meson Spectroscopy," Hadronic Journal, Vol. 1, 1978, p. 1591.

[15] V. P. Iyer and L. K. Sharma, "Heavy Meson Potentials," Indian Journal of Pure and Applied Physics, Vol. 20, 1982, pp. 322-324.

[16] E. Cuervo-Reyes, et al., "Hadron Spectra with a Nonrelativistic Model with Confining Harmonic Potential," Revista Brasilleria de Ensino de Fesica, Vol. 25, No. 1, 2003.

[17] K. J. Oyewumi and E. A. Bangudu, "Isotropic Harmonic Oscillator plus Inverse Quadratic Potential in N-Dimensional Space," The Arabian Journal for Science and En- 
gineering, Vol. 28, No. 2A, 2003, p. 173.

[18] C. Ansler, et al., "Particle Data Group," Physics Letters B, Vol. 667, 2008, Article ID: 010001. http://pdg.lbl.gov.

[19] F. F. Schoberl and W. Lucha, "Solving the Schroedinger Equation for Bound States with Mathematica," International Journal of Modern Physics C, Vol. 10, 1999, pp. 607-620.

[20] R. Van Royen and V. F. Weisskoph, "Vector Meson Leptonic Decay Width," Il Nuovo Cimento A, Vol. 50, No.
3, 1967, pp. 617-645. doi:10.1007/BF02823542

[21] G. R. Boroum and H. Abdolmalki, "Variational and Exact Solutions of the Wavefunction at Origin (WFO) for Heavy Quarkonium by Using a Global Potential," Physica Scripta, Vol. 80, 2009, Article ID: 065003.

[22] H. Chen, J. Zhang, Y.-B. Dong and P.-N. Shen, "Heavy Quarkonium Spectra in a Quark Potential Model," Chinese Physics Letters, Vol. 18, No. 12, 2001, p. 1558. doi:10.1088/0256-307X/18/12/305 